\title{
Broadband frequency response of a nonlinear resonator with clearance for energy harvesting
}

\author{
Krystian Łygas ${ }^{1, \star}$, Piotr Wolszczak ${ }^{1, \star \star}$, and Grzegorz Litak ${ }^{1,2, \star \star \star}$ \\ ${ }^{1}$ Department of Automation, Lublin University of Technology, Nadbystrzycka 36, PL-20-618 Lublin, Poland \\ ${ }^{2}$ Department of Process Control, AGH University of Science and Technology, Mickiewicza 30, PL-30-059 Krakow, Poland
}

\begin{abstract}
We examine the response of an inverted pendulum excited harmonically with amplitude limiters. Such a nonlinear resonator is suggested to work in the transduction of ambient vibration energy into the electrical power through bending of the moving beam. Using a multibody model we follow rotation and bending of the elastic beam and we provide the solutions of various frequencies. We found that the corresponding bending is the largest for subharmonic solutions of beam oscillations.
\end{abstract}

\section{Introduction}

Energy harvesting based on nonlinear resonators can provide more efficient ambient energy converters for various frequencies [1-4] comparing to linear devices [5]. The role of nonlinearities [6] is important not only in broadening of frequency in the main resonance region but also in involving the subharmonic and superharmonic frequencies in multiple solutions. For instance, possible solutions in double well system resonators [7] are related to the small amplitude single well solution and the large amplitude cross barrier solution. Especially, subharmonic solutions with a large amplitude are very useful for energy harvesting $[7,8]$. Following the concept of the inverted elastic pendulum which undergoes a bifurcation of single to double equilibria under load of a tip mass [9], we propose a system with the effect of clearance in the clamping.

In this note, we examine effect of clearance as a typical nonlinearity which can appear during exploitation. Furthermore, we show how the response of the system is changing with frequency sweep and discuss particular solutions.

\section{Dynamical Model}

Our model (Fig. 1) is based on a vertical flexible beam with a tip mas excited horizontally (see [9]). Instead of the usual clamping we consider the hinged connection with additional clearance. Because of contacts with limiters and motion around the hinged point the pendulum has two different possibilities to move: rotation and bending (see in Fig. 2). It possesses two

\footnotetext{
^e-mail: k.lygas@pollub.pl

${ }^{\star \star}$ e-mail: p.wolszczak@pollub.pl

$\star \star \star \star e-m a i l:$ g.litak@pollub.pl
}

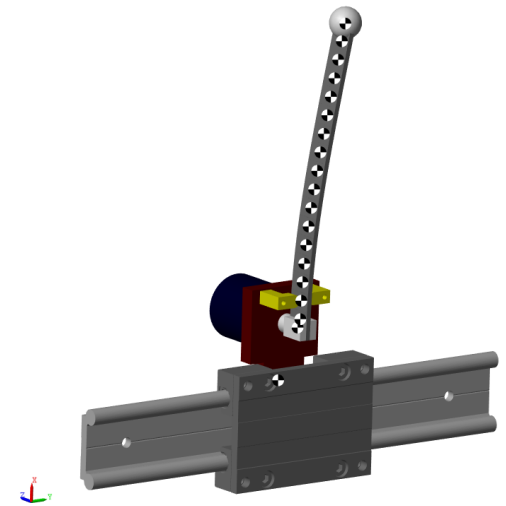

Figure 1. Scheme of the multibody system arrangements including the moving harmonically frame, planar inverted pendulum with amplitude limiters.

potential wells (Fig. 3) which could useful for a frequency broadband response [6]. Additional nonlinear effects are related to impacts into the amplitude limiters. This effect would be important for energy transduction $[12,13]$ as the final design is expected to be equipped in the piezoelectric patch along the bending beam surface (above the impacting region).

Flexible nonlinear beam systems have been already discussed in the previous works [14-16]. In particular, Tang and Ren [14] and Xu and Yu [15] examined a control algorithm to stabilize the pendulum in the upright position. On the other hand, Semenov et al. [16] discussed of a concept of elastic inverted Kapica like pendulum with the vertical excitation. Interestingly, their pendulum was also exposed on backlash following the hysteretic switching coupling. Later, Semenov at al. [17, 18] considered 


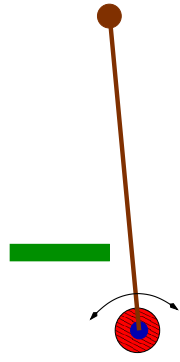

rotation

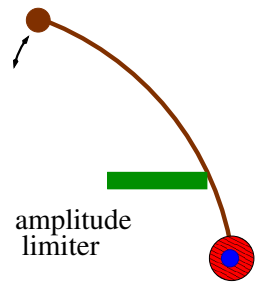

bending
Figure 2. Schematics of two different possible motions of the pendulum (marked by arrows).

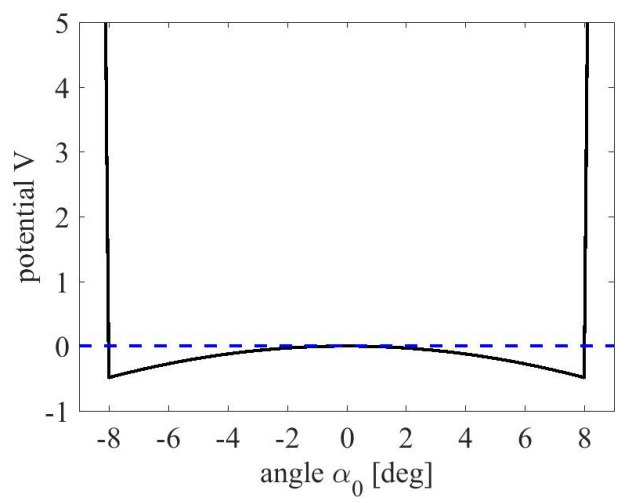

Figure 3. Normalized potential $V$ of the simplified rigid pendulum versus bearing angle $\alpha_{0}$.

also the horizontal excitation in the proposed system with a hysteretic coupling between the excitation frame and the inverted pendulum. We continue these studies on an inverted elastic pendulum by investigating capability of impacts for energy conversion. In our dynamical system the contact between amplitude limiters and the pendulum is defined in a different way (comparing to earlier proposals $[17,18]$ ).

The computational model is based on multistage inverted pendulum with 17 shorter and rigid beams and a tip mas. Kinetic, $T$, and potential, $V$, energies in the time intervals between impacts can be written as follows:

$$
\begin{aligned}
T & =\sum_{i=1}^{N} \frac{\Delta m_{i}\left(\mathbf{v}_{i}+\mathbf{v}_{i-1}\right)^{2}}{8} \\
& +\sum_{i=1} \frac{\Delta I_{i} \dot{\alpha}_{i}{ }^{2}}{2}+\frac{M \mathbf{v}_{N}^{2}}{2}, \\
V & =\sum_{i=1}^{N} \Delta m_{i} g \frac{\left(y_{i}+y_{i-1}\right)}{2} \\
& +\sum_{i=1}^{N} \frac{k\left(\alpha_{i}-\alpha_{i-1}\right)^{2}}{2}+M g y_{N},
\end{aligned}
$$

where $g=9.81 \mathrm{~m} / \mathrm{s}^{2}$ is the gravity acceleration, $M$ is a tip mass attached to the end node of beam element No. $N, \mathbf{v}_{i}=\left[x_{i}, y_{i}\right]$ are vectors denoting local connecting node velocities, $\alpha$ are angles between the neighbour short beam elements. All the elements have the same shape and sizes. Note that $\alpha_{0}$ is the bearing rotation and the assumed $v_{0}=\dot{x}_{0}$ of harmonic motion in the horizontal direction:

$$
x_{0}=A \sin (\omega t), \quad y_{0}=0,
$$

where $A$ is the amplitude while $\omega$ is the corresponding frequency of kinematic excitation. Furthermore, $\Delta m_{i}=\Delta m$ is mass of a short beam element while $\Delta I_{i}=\Delta I$ is the moment of inertia for defined elements, $y_{N}$ is the height on the tip mas $M$. Finally, $k$ is the local spring coefficient.
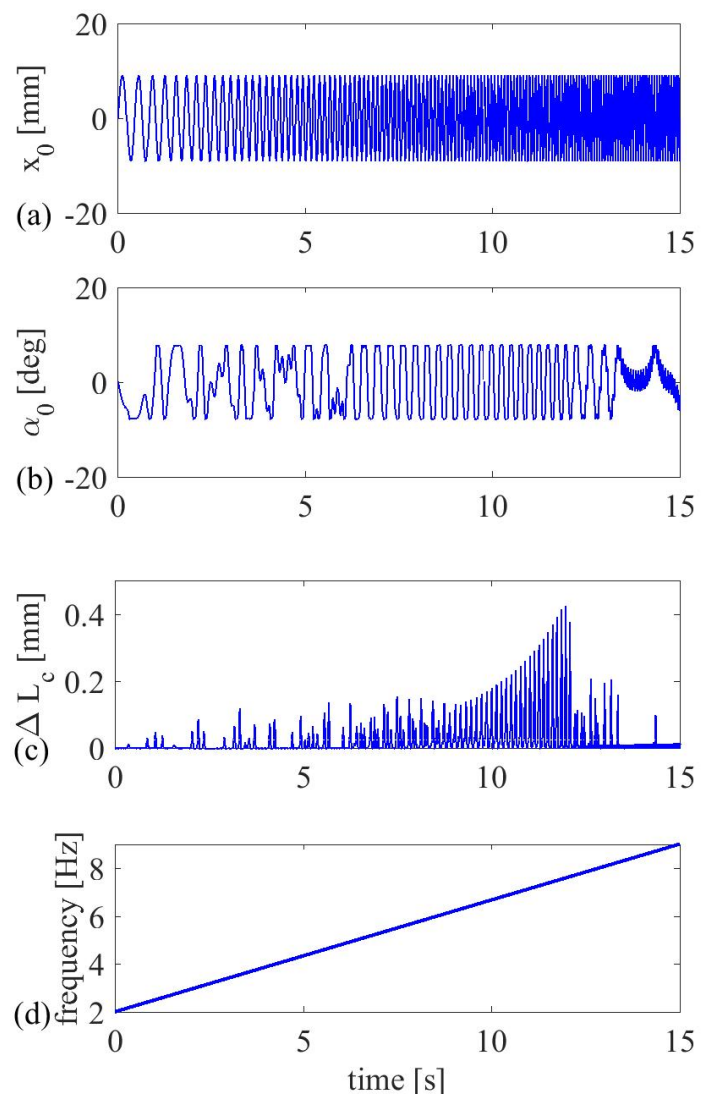

Figure 4. Frequency sweep is defined in the interval from 2 to $9 \mathrm{~Hz}$ (d). (a) shows the frame excitation $x$, (b) presents the $\alpha_{0}$ response, (c) is showing the time dependence $\Delta L_{c}$ as estimated bending in terms of the chord (connecting the beam ends) reduction.

The Lagrangian of the beam without further constraints (limiters) has the following form:

$$
\begin{array}{ll} 
& L\left(q_{1}, \ldots, q_{i}, \ldots, q_{N}, \dot{q}_{1}, \ldots, \dot{q}_{i}, \ldots, \dot{q}_{N}, t\right) \\
= & T\left(q_{1}, \ldots, q_{i}, \ldots, q_{N}, \dot{q}_{1}, \ldots, \dot{q}_{i}, \ldots, \dot{q}_{N}, t\right) \\
-\quad & V\left(q_{1}, \ldots, q_{i}, \ldots, q_{N}, \dot{q}_{1}, \ldots, \dot{q}_{i}, \ldots, \dot{q}_{N}, t\right),
\end{array}
$$

where the generalized coordinates this multistage inverted pendulum $q_{i} \in\left\{\alpha_{1}, \ldots, \alpha_{n}\right\}$ and their corresponding velocities $\dot{q}_{i} \in\left\{\dot{\alpha}_{1}, \ldots, \dot{\alpha}_{n}\right\}$. Consequently, 
the general equation of motions for such a kinetically forced inverted pendulum can be written as:

$$
\frac{d}{d t}\left(\frac{\partial L}{\partial \dot{q}_{j}}\right)-\frac{\partial L}{\partial q_{j}}=Q_{j}+Q_{n}^{i m p}
$$

(a)
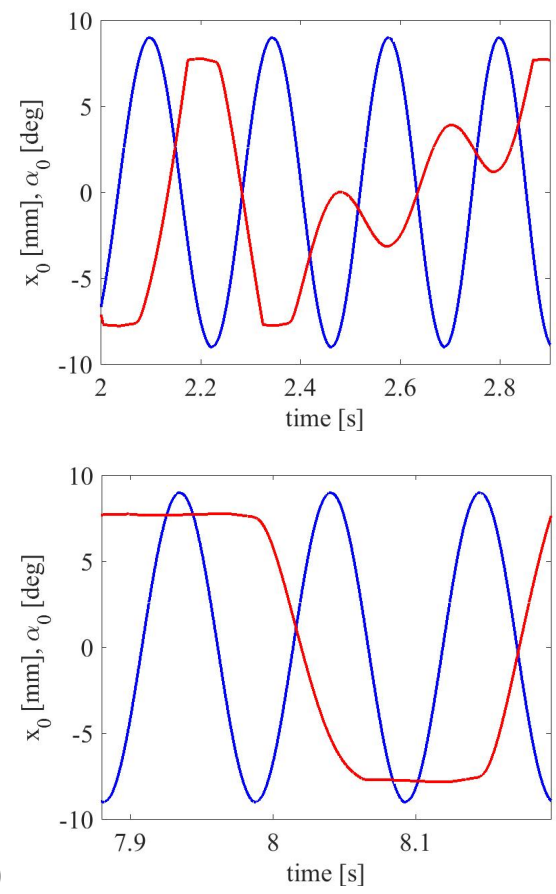

Figure 5. Waveform responses based on the frequency sweep results (Fig. 4), where blue line denotes a frame displacement, $x_{0}$ and red line the angular $\alpha_{0}$ displacement of bearings, $\alpha_{0}$. Responses are ploted for different excitation frequencies $f=3.1,5.7,6.7,7.8,8.8 \mathrm{~Hz}$, for cases (a)-(e), respectively.

The generalized forces $Q_{j}$ appearing in the above equation are defined:

$$
Q_{j}=-\beta\left(\dot{\alpha}_{j}-\dot{\alpha}_{j-1}\right)-\beta\left(\dot{\alpha}_{j}-\dot{\alpha}_{j+1}\right),
$$

while the additional generalized force $Q_{n}^{i m p}$ is caused by an inelastic contact to one of amplitude limiters with local contact elasticity and damping properties (see Tab. 1 for parameter values and Figs. 1 and 2 for view). They are attached to the beam elements and cause the whole beam to bounce on the amplitude limiters.

\section{Simulation results}

Using the equations of motion (Eqs. 5-6) and parameters of the system are presented in Tab. 1 we performed simulations. The calculations of the dynamical response of the introduced pendulum-impact model were performed by using Maltab-Simulink and Simscape Multibody with Flexible Beam and Contact Forces libraries.

The frequency sweep results for of calculation are presented in Fig. 4. Here, in the time interval of
$15 \mathrm{~s}$ we changed steadily the excitation frequency in the range of 2-9 Hz. Interestingly, the system behavior shows a number of bifurcations. In the low frequency the response is nonperiodic while in the range $f \in[5-7]$ subharmonic 3 dominate. For larger frequency we found subharmonic 5 and transition to nonperiodic behaviours.

(c)
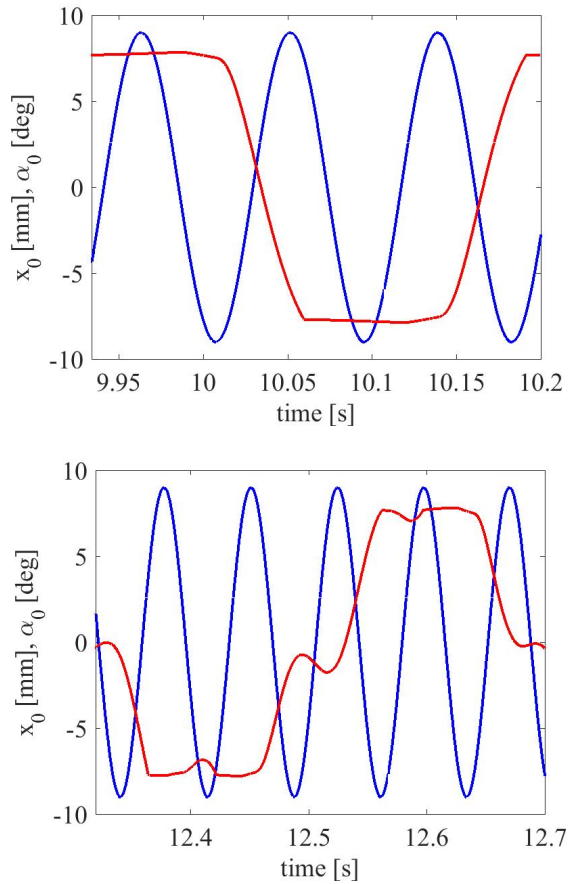

(d)

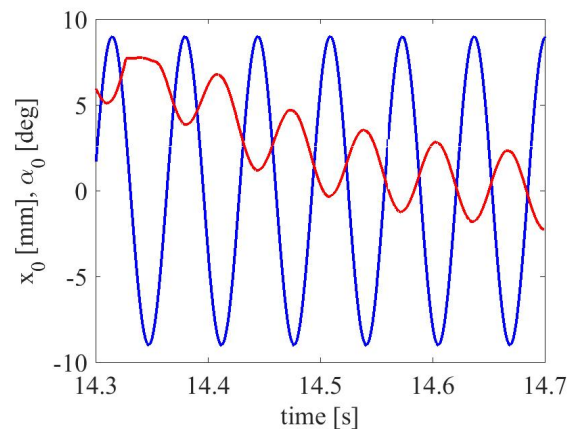

Figure 5. Continuation.

For better clarity it the characteristic waveforms are ploted also in Fig. 5. Note that Figs. 5a and e shows clearly intervals of nonperiodic responses in the small $(f=3.1 \mathrm{~Hz})$ and high $(\mathrm{f}=8.8 \mathrm{~Hz})$ frequencies from the interval $f \in[2 \mathrm{~Hz}, 9 \mathrm{~Hz}]$ (Fig. 4), while in the middle frequencies $(\mathrm{f}=5.7$ and $6.7 \mathrm{~Hz}$ ) correspond to a subharmonic solution of period 3 with respect to the excitation period (see blue and red lines in Figs. 5b and c). Finally, at the excitation frequency $f=7.8 \mathrm{~Hz}$ (Fig. 5d) a subharmonic solution of period 5 is present. The periodic waveforms (shown in Figs. 5b-d) are characterized by the large amplitude oscillations with passing through the potential barrier (Fig. 3). Interestingly, the maximum of bending response (Fig. 4c) is reached for subharmonic solution of period 3 . 
Table 1. Material and geometrical properties of beam and tip mass.

\begin{tabular}{|c|c|}
\hline ultimate tensile strength (ASTM D638) & $7.5 \mathrm{MPa}$ \\
Young Modulus (ASTM D638) & $350 \mathrm{MPa}$ \\
elongation at break (ASTM D638) & $3-5 \%$ \\
typical parts density & $0.85 \mathrm{~g} / \mathrm{cm}^{3}$ \\
beam dimensions (width x thickness x length) & $17 \times 3 \times 195 \mathrm{~mm}$ \\
natural vibration of elastic beam & first mode above $9 \mathrm{~Hz}$ \\
tip mass & $1.129 \mathrm{~g}$ \\
distance between limiters & $12 \mathrm{~mm}$ \\
contact stiffness & $10^{4} \mathrm{~N} / \mathrm{m}$ \\
contact damping & $10^{2} \mathrm{Ns} / \mathrm{m}$ \\
\hline
\end{tabular}

\section{Conclusions}

The obtained results show that the system response of elastic inverted pendulum with amplitude limiters of fixed distance has a nonlinear features of double well oscillator with soft impacts. For the fixed distance between the amplitude limiters excitation, excitation frequency was changed quasi-statically during simulation. Examining the response wave forms one can notice that there are both periodic and nonperiodic responses. Periodic responses of the large amplitude appeared to be subharmonic solutions of the period 3 and 5. Especially, the subharmonic solution of excitation period 3 was interesting for energy harvesting showing simultaneously the capability of fairly high bending (Fig. 4c). This solution is consistent with the recent results by Huguet et al. [8] and Syta et al. [7] where the subharmonic solutions were present. Here, the beam deflection response is formed in a sequence of peaks depending on impacts in the chosen system geometry and dynamical conditions. Such peaks will produce an electrical impulses [19] on the system once it is adapted to energy harvesting by the piezoelectric patches. For energy harvesting, both maximal values and higher frequency are important, Therefore harmonic and subharmonic solution of period 3 and those of period 5 with smaller bending, or noperiodic solutions can be of importance. Finally, we advocate the application of the proposed mechanical system as a resonator in a broadband frequency energy harvester including the subharmonic and also nonperiodic solutions.

\section{References}

[1] P.D. Mitcheson, E.M. Yeatman, G.K. Rao, A.S. Holmes, T.C. Green, Proceedings of the IEEE 96, 1457 (2008)

[2] R.L. Harne, K.W. Wang, Smart Mat. Struct. 22, 023001 (2013)

[3] S.P. Pellegrini, N. Tolou, M. Schenk, J.L. Herder, J. Intell. Mater. Syst. Struct. 24, 1303 (2013)
[4] J. Twiefel, H. Westermann, J. Intell. Mater. Syst. Struct. 24, 1291 (2013)

[5] S.P. Beeby, M.J. Tudor, N.M. White, Meas. Sci. Technol. 17, R175 (2006)

[6] M.F. Daqaq, R. Masana, A. Erturk, D.D. Quinn, Applied Mechanics Reviews 66, 040801 (2014)

[7] A. Syta, G. Litak, M.I. Friswell, S. Adhikari, Eur. Phys. J. B 89, 99 (2016)

[8] T. Huguet, A. Badel, A., M. Lallart, Appl. Phys. Lett. 111, 173905 (2017)

[9] M.I. Friswell, S.F. Ali, S. Adhikari, A.W. Lees, O. Bilgen, G. Litak, J. Intell. Mater. Syst. Struct. 23, 1505 (2012)

[10] A. Erturk, J. Hoffmann, D.J. Inman, Appl. Phys. Lett. 94, 254102 (2009)

[11] F. Cottone, H. Vocca, L. Gammaitoni, Phys. Rev. Lett. 102, 080601 (2009)

[12] S. Stoykov, E. Manoach, G. Litak, Eur. Phys. J. Special Topics 224, 2755 (2015)

[13] M. Borowiec, G. Litak, S. Lenci, Inter. J. Struct. Stab. Dynamics 14, 1440020 (2014)

[14] J. Tang, G. Ren, Tsinghua Science and Technology 14, 22 (2009)

[15] C. Xu, X. Yu, J. Contr. Theory Applic. 3, 281 (2004)

[16] M.E. Semenov, P.A. Meleshenko, A.M. Solovyov, A.M. Semenov, Hysteretic nonlinearity in inverted pendulum problem, in Structural Nonlinear Dynamics and Diagnosis, edited by M. Belhaq (Springer, Cham, 2012), pp. 463-506

[17] M.E. Semenov, A.M. Solovyev, Stabilization of elastic pendulum with hysteresis, in International Conference Information Technology and Nanotechnology, CEUR Workshop Proceedings DOI: 10.18287/1613-0073-2016-1638-650-657 (Samara, 2016), pp. 650-657

[18] M.E. Semenov, A.M. Solovyov, P.A. Meleshenko, Nonlin. Dynamics 82, 677 (2015)

[19] S. Adhikari, M.I. Friswell, G. Litak, H.H. Khodaparast, Smart Mater. Struc. 25, 065009 (2016) 